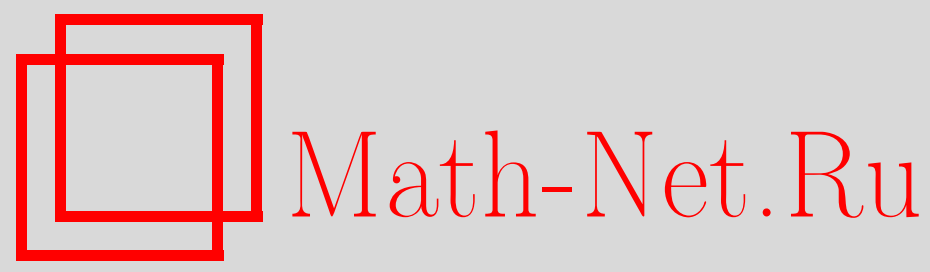

В. Я. Эйдерман, Мера Хаусдорфа и емкость, ассоциированная с потенциалами Коши, Матем. заметки, 1998, том 63, выпуск 6, 923-934

DOI: https://doi.org/10.4213/mzm1363

Использование Общероссийского математического портала Math-Net.Ru подразумевает, что вы прочитали и согласны с пользовательским соглашением http://www . mathnet.ru/rus/agreement

Параметры загрузки:

IP: 54.210 .77 .194

26 апреля 2023 г., 13:09:16

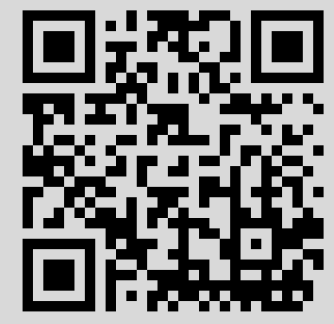




\section{МЕРА ХАУСДОРФА И ЕМКОСТЬ, АССОЦИИРОВАННАЯ С ПОТЕНЦИАЛАМИ КОШИ}

\section{В.Я. Эйдерман}

Мы изучаем связь меры Хаусдорфа $\Lambda_{h}(E)$ множества $E \subset \mathbb{C}$ с аналитической емкостью $\gamma(E)$ и емкостью $\gamma^{+}(E)$, порождаемой потенциалами Коши с неотрицательными мерами. Показываем, что если интеграл $\int_{0} t^{-3} h^{2}(t) d t$ расходится и $h$ удовлетворяет дополнительному условию регулярности, то найдется плоское канторово множество $E$, для которого $\Lambda_{h}(E)>0$, но $\gamma^{+}(E)=0$. Доказательство основано на оценке величины $\gamma^{+}\left(E_{n}\right)$, где $E_{n}$ - множество, возникающее на $n$-м шаге построения плоского канторова множества.

Библиография: 17 названий.

Введение. В данной работе мы изучаем связь меры Хаусдорфа $\Lambda_{h}(E)$ множества $E \subset \mathbb{C}$ с аналитической емкостью $\gamma(E)$ и емкостью $\gamma^{+}(E)$, порождаемой потенциалами Коши с неотрицательными мерами. Приведем необходимые определения.

Функцию $h(t)$ будем называть измеряющей, если она непрерывна, возрастает при $t \geqslant 0$ и $h(0)=0$. Пусть $E \subset \mathbb{C}$ - ограниченное множество. Мера Хаусдорфа $\Lambda_{h}(E)$ определяется равенством

$$
\Lambda_{h}(E)=\lim _{\rho \rightarrow 0} \inf _{r_{i} \leqslant \rho} \sum_{i} h\left(r_{i}\right),
$$

где нижняя грань берется по всем покрытиям множества $E$ не более чем счетными наборами шаров с радиусами $r_{i}$, непревосходящими $\rho$. Аналитической емкостью компакта $E \subset \mathbb{C}$ назьвается величина

$$
\gamma(E)=\sup \lim _{z \rightarrow \infty}|z f(z)|
$$

где верхняя грань берется по функциям $f$, аналитическим и ограниченным по модулю единищей в $\mathbb{C} \backslash E, f(\infty)=0$. Понятие аналитической емкости было введено Л. Альфорсом (1947) в связи с задачей об устранимых особенностях ограниченных аналитических функций: множество $E$ устранимо для указанного класса функций тогда и только тогда, когда $\gamma(E)=0$. Ряд свойств величины $\gamma(E)$ приводится, например, в [1]. Определим емкость $\gamma^{+}(E)$ :

$$
\gamma^{+}(E)=\sup _{\mu} \int_{E} d \mu
$$

Работа выполнена при частичной поддержке Королевской Академии наук Швеции, Международного научного фонда, грант МВ 6000, и Госкомвуза РФ, грант № 95-0-1.7-52. 
где мера $\mu$ такова, что

$$
\left|\int_{E} \frac{d \mu(\zeta)}{\zeta-z}\right|<1, \quad z \in \mathbb{C} \backslash E, \quad \operatorname{supp} \mu \subseteq E, \quad d \mu \geqslant 0 .
$$

Емкости $\gamma$ и $\gamma^{+}$, по-видимому, тесно связаны. Неизвестно, существует ли компакт $E$, для которого $\gamma^{+}(E)=0$, но $\gamma(E)>0$. Величина $\gamma^{+}$изучалась, например, в [2]. Мы будем рассматривать также ньютонову емкость

$$
C(E)=\sup _{\mu} \int_{E} d \mu,
$$

где мера $\mu$ такова, что

$$
\int_{E} \frac{d \mu(\zeta)}{|\zeta-z|}<1, \quad z \in \mathbb{C}, \quad \operatorname{supp} \mu \subseteq E, \quad d \mu \geqslant 0 .
$$

(Здесь достаточно потребовать ограниченности потенциала лишш на множестве $E$, по принципу максимума он будет ограничен всюду.) Непосредственно из определений следует, что

$$
C(E) \leqslant \gamma^{+}(E) \leqslant \gamma(E) .
$$

В силу известной теоремы Пенлеве

a) если $\gamma(E)>0$, то $\Lambda_{h}(E)>0$ при

$$
h(t)=t .
$$

С другой стороны, по теореме

б) если

$$
\int_{0} \frac{h(t)}{t^{2}} d t<\infty
$$

$$
\text { и } \left.\Lambda_{h}(E)>0 \text {, то } C(E)>0 \text { (см. }[3, \text { с. } 35],[1, \text { с. } 73]\right) \text {, а значит, ввиду }(1) \text { и } \gamma(E)>0 \text {. }
$$

Возникает вопрос: можно ли уменьшить зазор между условиями $(2)$ и $(3)$ на $h$ в утверждениях а) и б)? Пример $E=[a, b]$ показывает, что в первом из них нельзя взять $h(t)=o(t)$, $t \rightarrow 0+$. Естественно в связи с этим исследовать необходимость условия (3). (Для емкостей, порождаемых потенциалами с положительньми ядрами, аналогичный вопрос изучался автором в [4], [5].) В случае $h(t)=t$ А. Г. Витушкиным [6], затем Дж. Гарнеттом [7], были построены примеры компактов $E$, для которых $\Lambda_{h}(E)>0$, но $\gamma(E)=0$. Более широкий класс таких множеств описан в [8]. В [9, с. 151] Л. Д. Иванов выдвинул следующую гипотезу.

ГИПотеЗА 1. Для всякой измеряющей функиии $h$ такой, что

$$
\int_{0} \frac{h(t)}{t^{2}} d t=\infty
$$

найдется компакт $E$ такой, что $\Lambda_{h}(E)>0 u \gamma(E)=0$.

В [9, с. 152-153] автор отметил, что гипотеза 1 не верна без дополнительных условий регулярности на функцию $h$. В $[1$, с. 94 , теорема 2.7$]$ также формулируется утверждение, аналогичное гипотезе 1 , которое доказьвается при дополнительном условии: функция $a(t)=t h^{\prime}(t) / h(t)$ возрастает - сравнением величин $C(E)$ и $\gamma(E)$ для плоских канторовых множеств $E$. 
Зададим последовательность положительных чисел $\left\{l_{j}\right\}_{j=0}^{\infty}$ такую, что $2 l_{j}<l_{j-1}$, $j \geqslant 1$. Возьмем некоторьй отрезок $E^{(0)} \subset \mathbb{R}^{1}$ длины $l_{0}$. От конщов этого отрезка внутрь $E^{(0)}$ отложим отрезки $E_{j}^{(1)}, j=1,2$, длины $l_{1}$. Положим $E^{(1)}=E_{1}^{(1)} \cup E_{2}^{(1)}$. Далее, на каждом отрезке $E_{j}^{(1)}$ аналогичньм образом расположим по два отрезка $E_{j, i}^{(2)}$ длины $l_{2}$. Объединение полученных четырех отрезков $E_{j, i}^{(2)}$ обозначим через $E^{(2)}$. Продолжая этот процесс, получим последовательность множеств $E^{(n)}$, причем $E^{(n)}$ состоит из $2^{n}$ отрезков длины $l_{n}$. Положим $E_{n}=E^{(n)} \times E^{(n)}$. Очевидно, $E_{n}$ состоит из $4^{n}$ квадратов, которые обозначим через $E_{n, j}$. Наконец, положим $E=\bigcap_{n=0}^{\infty} E_{n}$. Множество $E=E\left(\left\{l_{j}\right\}\right)$ назьвается плоским канторовым множеством.

В $[1$, с. 88 , теорема 2.2$]$ утверждается, что если $E=E\left(\left\{l_{j}\right\}\right)$ и последовательность $\left\{l_{j+1} / l_{j}\right\}$ не возрастает, то $C(E)=0 \Longleftrightarrow \gamma(E)=0$. В [9, с. 153] указано, что в доказательстве этого утверждения есть пробел. В [10, теорема 3.9] П. Маттила показал, что и само утверждение неверно. А именно, если последовательность $\left\{l_{j+1} / l_{j}\right\}$ невозрастает, а измеряющая функция $h$ такова, что $h\left(l_{j}\right)=4^{-j}, j \geqslant 0$, и

$$
\int_{0} \frac{h^{2}(t)}{t^{3}} d t<\infty
$$

то $\gamma\left(E\left(\left\{l_{j}\right\}\right)\right)>0$. (Более общие результаты Маттилы приводятся в п. 2.) Это утверждение означает, что

$$
\sum_{j=1}^{\infty}\left(\frac{4^{-j}}{l_{j}}\right)^{2}<\infty \Longrightarrow \gamma\left(E\left(\left\{l_{j}\right\}\right)\right)>0
$$

поскольку при $h\left(l_{j}\right)=4^{-j}$ сходимость интеграла в (4) равносильна сходимости ряда в (5) (см. ниже неравенства (21); нетрудно провести и обратные оценки). В то же время известно (см., например, [3, с. 38]), что

$$
\sum_{j=1}^{\infty} \frac{4^{-j}}{l_{j}}<\infty \Longleftrightarrow C\left(E\left(\left\{l_{j}\right\}\right)\right)>0 .
$$

В [10] обсуждается обратимость теоремы 3.9, но этот вопрос к настоящему времени остается открытым.

В данной работе мы рассматриваем широкий класс "регулярных" множеств (включающий, в частности, канторовы множества). Мы показываем, что при сравнении емкостей $\gamma, \gamma^{+}$и меры Хаусдорфа таких множеств критическую роль играет сходимость интеграла в (4), а не в (3). Легко убедиться, что из (3) следует (4), обратное неверно. Таким образом, гипотеза 1 и теорема 2.7 из [1] для рассматриваемого класса множеств неверны (в силу теоремы 3.8 из [10]; подробнее см. п. 1 и п. 2). Полученные результаты, относящиеся к емкости $\gamma^{+}$, в некотором смысле окончательны. Важную роль в доказательстве будет играть понятие кривизны меры, введенное М. С. Мельниковым в [11].

1. Основные результаты. В дальнейшем допускается использование одинаковых обозначений для различньх постоянных.

Пусть $h$ - измеряющая функция. Назовем компакт $E \subset \mathbb{C} h$-регулярным, если сушествуют постоянные $0<c_{1}<c_{2}<\infty$ и $r_{0}>0$ такие, что

$$
c_{1} h(r) \leqslant \Lambda_{h}(E \cap B(x, r)) \leqslant c_{2} h(r), \quad x \in E, \quad 0<r \leqslant r_{0}
$$


(здесь и далее $B(x, r)=\{z \in \mathbb{C}:|z-x|<r\})$. Например, если $E=E\left(\left\{l_{j}\right\}\right)$-канторово множество и $h$ такова, что $h\left(l_{j}\right)=4^{-j}$, то $E$ является $h$-регулярным. В случае $h(r)=r$ понятие $h$-регулярности совпадает с регулярностью в смысле Альфорса-Давида (см., например, [12, с. 104]).

Маттила [10] установил, что условие (4) является достаточным для положительности емкости $\gamma \quad h$-регулярных множеств. Для полноты изложения мы приведем оригинальные формулировки из [10] в п. 2, а теперь сформулируем два прямых следствия этих результатов (теоремы 1 и 2), необходимых для изучаемой нами задачи.

ТЕОРема 1. Пусть компакт $E \subset \mathbb{C}$ является h-регулярным для такой измеряющей функции $h$, которая удовлетворяет условию (4) и условию удвоения $h(2 r) \leqslant c h(r), r>0$, с некоторой постоянной $c>1$. Тогда $\gamma^{+}(E)>0$.

По-видимому, условие удвоения и первое неравенство в (6) могут быть опущены. Справедлива следующая теорема, в которой эти условия отсутствуют, но вместо положительности емкости $\gamma^{+}$утверждается лиш положительность $\gamma$.

Teорема 2. Пусть $\Lambda_{h}(E)>0, \Lambda_{h}(E \cap B(x, r)) \leqslant c_{2} h(r), x \in E$, әде $0<r \leqslant r_{0} u$ постоянная $c_{2}<\infty$. Пусть выполнено условие (4). Тогда $\gamma(E)>0$.

Из теоремы 1 вытекает

СЛЕДСТВИЕ 1. Пусть $E=E\left(\left\{l_{j}\right\}\right)$ - плоское канторово множество, а измеряющая функиия $h$ такова, что $h\left(l_{j}\right)=4^{-j}, j \geqslant 0$, и выполнено условие (4). Тогда $\gamma^{+}(E)>0$.

Следствие 1 усиливает теорему 3.9 из [10]. Из следствия 1 вытекает импликация

$$
\sum_{j=1}^{\infty}\left(\frac{4^{-j}}{l_{j}}\right)^{2}<\infty \Longrightarrow \gamma^{+}\left(E\left(\left\{l_{j}\right\}\right)\right)>0
$$

причем без дополнительного условия невозрастания последовательности $\left\{l_{j+1} / l_{j}\right\}$.

Рассмотрим теперь вопрос о необходимости условия (4). Основными результатами данной работы являются теоремы 3 и 4.

ТЕОрема 3. Пусть измеряющая функиия $h(t)$ удовлетворяет условиям

$$
\begin{gathered}
\int_{0} \frac{h^{2}(t)}{t^{3}} d t=\infty, \\
h\left(t_{1}\right) t_{1}^{-\alpha} \geqslant b h\left(t_{2}\right) t_{2}^{-\alpha}, \quad 0<t_{1} \leqslant t_{2} \leqslant r_{0},
\end{gathered}
$$

с некоторыми постоянными $r_{0}>0, b \in(0,1) u 0<\alpha<\log _{3} 4$. Тогда найдется $h$-регулярный компакт $E \subset \mathbb{C}$ такой, что $\gamma^{+}(E)=0$.

Возможно, условие (8) можно ослабить. Но его нельзя отбросить: нетрудно показать, что найдется такая измеряющая функция $h(t)$, для которой вьполнено условие $(7)$ и $\Lambda_{h}(\mathbb{C})=0$, т.е. $h$-регулярных компактов не существует (обоснование этого факта основано на идее доказательства предложения 3.3 из [5]). Заметим, что условие регулярности (8) не является слишком ограничительным; ему удовлетворяет широкий класс измеряющих функций, содержащий и функции, для которых вьполнено (4), и функции со свойством (7).

Теорема 3 выводится из следующей оценки для множеств $E_{n}$, состоящих из $4^{n}$ квадратов и описанных во введении. 
Tеорема 4. Пусть плоское канторово множество $E=E\left(\left\{l_{j}\right\}\right)$ таково, что $l_{j+1} / l_{j} \leqslant \lambda<1 / 3$. Тогда найдется постоянная $c$, зависящая только от $\lambda$, такая, чmo

$$
\gamma^{+}\left(E_{n}\right)<c\left(\sum_{j=1}^{n}\left(\frac{4^{-j}}{l_{j}}\right)^{2}\right)^{-1 / 2}, \quad n \geqslant 1 .
$$

СлЕДСТвИЕ 2. Пусть $l_{j+1} / l_{j} \leqslant \lambda<1 / 3$. Тогда

$$
\sum_{j=1}^{\infty}\left(\frac{4^{-j}}{l_{j}}\right)^{2}<\infty \Longleftrightarrow \gamma^{+}\left(E\left(\left\{l_{j}\right\}\right)\right)>0 .
$$

Оценку (9) дополняет следующее

ПРЕДЛОЖЕНИЕ 1. Найдется абсолютная постоянная $Q>0$ такая, что

$$
\gamma\left(E_{n}\right)>Q\left(\sum_{j=1}^{n}\left(\frac{4^{-j}}{l_{j}}\right)^{2}\right)^{-1 / 2}, \quad n \geqslant 1 .
$$

При $l_{j}=4^{-j}$ получаем $\gamma\left(E_{n}\right)>Q / \sqrt{n}$, что подтверждает гипотезу 1 из [13]: если $l_{j}=4^{-j}$, то $\gamma\left(E_{n}\right)=p_{n} / n$, где $p_{n} \rightarrow \infty$ при $n \rightarrow \infty$. Для сравнения приведем оценку величины $C\left(E_{n}\right)$, вытекающую из доказательства лемм 1.3 и 1.5 работы [5]:

$$
A_{1}\left(\sum_{j=1}^{n} \frac{4^{-j}}{l_{j}}\right)^{-1}<C\left(E_{n}\right)<A_{2}\left(\sum_{j=1}^{n} \frac{4^{-j}}{l_{j}}\right)^{-1}, \quad n \geqslant 1,
$$

где $A_{1}, A_{2}$ - абсолютные постоянные.

Неравенства (9) и (10) позволяют выдвинуть следующую гипотезу.

ГипотеЗА 2. Пусть $l_{j+1} / l_{j} \leqslant \lambda<1 / 3$. Найдутся постоянные $Q_{1}, Q_{2}$, зависящие только от $\lambda$, такие, что

$$
Q_{1}\left(\sum_{j=1}^{n}\left(\frac{4^{-j}}{l_{j}}\right)^{2}\right)^{-1 / 2}<\gamma^{+}\left(E_{n}\right) \leqslant \gamma\left(E_{n}\right)<Q_{2}\left(\sum_{j=1}^{n}\left(\frac{4^{-j}}{l_{j}}\right)^{2}\right)^{-1 / 2}, \quad n \geqslant 1 .
$$

Заметим, что единственная известная автору оценка для $\gamma\left(E_{n}\right)$ относится к случаю $l_{j}=4^{-j}$ и принадлежит Т. Мураи [14]: $\gamma\left(E_{n}\right) \leqslant c / \log n$. Неравенство (9) в этом случае дает более слабую оценку $\gamma^{+}\left(E_{n}\right) \leqslant c / \sqrt{n}$. Несмотря на то что функция Альфорса для $E_{n}$ представима в виде потенциала Коши, мера $\mu$ в этом представлении, по-видимому, комплексная.

\section{2. Доказательства результатов.}

ДоКАЗАТЕЛЬСТво ТЕОРЕМЫ 2. В работе [10] установлена следующая теорема.

Tеорема 5 [10, с. 202, теорема 3.8]. Пусть $E$ - такой компакт в $\mathbb{C}$, для которого найдутся ненулевая борелевская мера, сосредоточенная на Е, и измеряющая функиия $h$ такие, что выполнено (4) $u$

$$
\mu(B(z, r)) \leqslant h(r), \quad z \in \mathbb{C}, \quad r>0 .
$$

Тогда $\gamma(E)>0$.

Из справедливости неравенства $\Lambda_{h}(E \cap B(x, r)) \leqslant c_{2} h(r)$ при $0<r \leqslant r_{0}$ следует, что оно вьполняется и при всех $r>0$ (возможно, с большей постоянной $c_{3}$ вместо $c_{2}$ ). Возьмем теперь в качестве $\mu$ меру $\mu(F)=c_{3}^{-1} \Lambda_{h}(E \cap F)$. Поскольку (11) выполнено, теорема 2 сразу следует из теоремы 5 . 
ДоКАЗАТЕЛЬСТво ТЕОРЕмЫ 1. Теорема 5 вьводится в [10] из теоремы 3 Мельникова [11] (будет приведена ниже). Маттила заметил, что существует и другой способ установить аналогичное утверждение, но при дополнительном условии удвоения на меpy $\mu$ :

$$
\mu(B(z, 2 r)) \leqslant c \mu(B(z, r)), \quad z \in \operatorname{supp} \mu, \quad r>0 .
$$

Теорема 6 [10, с. 202, теорема 3.7]. Предположим, что $h$ и $\mu$ удовлетворяют условиям (4), (11) и (12). Тогда операторь

$$
\mathscr{C}_{\mu, \varepsilon}(g)=\int_{|\zeta-z|>\varepsilon} \frac{g(\zeta)}{\zeta-z} d \mu(\zeta): L^{2}(\mu) \rightarrow L^{2}(\mu)
$$

ограничены, причем равномерно относительно $\varepsilon$.

Более того, Маттила указал, что методами, изложенными в [12, гл. 7], из теоремы 6 выводится

ПРЕДЛОЖЕНИЕ 2. Если на компакте $E \subset \mathbb{C}$ существует ненулевая борелевская мера $\mu$, удовлетворяющая условиям (4), (11) $u(12)$, то $\gamma(E)>0$.

Заметим, что методы из [12] позволяют вывести из теоремы 6 более сильное чем предложение 2

ПРЕДЛОЖЕНИЕ 3. Величину $\gamma(E)$ можно заменить на $\gamma^{+}(E)$.

Далее, как и при доказательстве теоремы 2 , возьмем $\mu(F)=c_{3}^{-1} \Lambda_{h}(E \cap F)$. Условия (4), (11) вьполнены, а справедливость неравенства (12) следует из (6) и условия $h(2 r) \leqslant c h(r)$. Теорема 1 доказана.

ДоКАЗАТЕЛЬСТВо СЛЕДСТВИЯ 1. Чтобы установить $h$-регулярность множества $E$, воспользуемся следующей оценкой величины $\Lambda_{h}(E)$ :

$$
Q_{1} L \leqslant \Lambda_{h}(E) \leqslant Q_{2} L, \quad \text { где } L=\liminf _{j \rightarrow \infty} 4^{j} h\left(l_{j}\right),
$$

а $Q_{1}$ и $Q_{2}$ - абсолютные постоянные. Оценка (13) непосредственно следует из теоремы 3.1 работы [4], если положить $k_{j}=2, M_{1}=2, M_{2}=1$. Теперь заметим, что $\Lambda_{h}(E \cap B(x, r)) \asymp \Lambda_{h}\left(E^{r}\right)$ (т.е. отношение этих величин заключено между двумя абсолютньпи положительными постоянными). Здесь $E^{r}=E\left(\left\{l_{j}^{r}\right\}_{j=0}^{\infty}\right)$ - канторово множество с $l_{j}^{r}=l_{j+j_{0}}$, а $j_{0}$ определяется неравенствами $l_{j_{0}+1} \leqslant r<l_{j_{0}}$. Поскольку

$$
h\left(l_{j}^{r}\right)=4^{-\left(j+j_{0}\right)}=4^{-j} h\left(l_{j_{0}}\right) \asymp 4^{-j} h(r),
$$

то следствие 1 вытекает из оценок (13), примененных к множеству $E^{r}$.

Проверим условие удвоения. Пусть $l_{i+1} \leqslant r<l_{i}$. Тогда $l_{i+1}<2 r<l_{i-1}$. Поэтому

$$
h(2 r)<4^{-i+1}=16 \cdot 4^{-i-1}=16 h\left(l_{i+1}\right) \leqslant 16 h(r) .
$$

Теперь неравенство $\gamma^{+}(E)>0$ следует из теоремы 1 .

Положим

$$
\mathscr{C} \mu(z)=\int_{\mathbb{C}} \frac{d \mu(\zeta)}{\zeta-z}
$$


Лемма 1. Предположим, что последовательность $\left\{l_{j}\right\}$ удовлетворяет условию $l_{j+1} / l_{j} \leqslant \lambda<1 / 2, j \geqslant 0, u E_{n}-$ множество, описанное во введении. Пусть $\mu$ - произвольная (вообще говоря, комплексная) мера, сосредоточенная на $E_{n}$, такая, что $|\mathscr{C} \mu(z)|<1, z \in \mathbb{C} \backslash E_{n}$. Обозначим через $\nu$ меру, которая сосредоточена на $E_{n}$, причем $\nu\left(E_{n, j}\right)=\mu\left(E_{n, j}\right)$ для любых $n, j$, и на каждом квадрате $E_{n, j}$ распределена равномерно. Тогда $|\mathscr{C} \nu(z)|<A, z \in \mathbb{C}$, где постоянная $A$ зависит только от $\lambda$.

ДокАЗАТЕЛьСтво. Воспользуемся идеей доказательства леммы 2.4 из [1, с. 90]. Положим

$$
\begin{gathered}
f(z)=\mathscr{C} \mu(z), \quad f_{n, j}(z)=-\frac{1}{2 \pi i} \int_{\gamma_{n, j}} \frac{f(\zeta) d \zeta}{\zeta-z}, \\
\varphi_{n, j}(z)=\int_{E_{n, j}} \frac{d \nu(\zeta)}{\zeta-z}, \quad a_{n, j}=\mu\left(E_{n, j}\right),
\end{gathered}
$$

где $\gamma_{n, j}$ - контур, однократно обходящий квадрат $E_{n, j}$ и не содержащий точку $z$ внутри себя. Как и в лемме 2.3 из $\left[1\right.$, с. 90], $\left|f_{n, j}(z)\right|<c_{1}, z \in \mathbb{C} \backslash E_{n, j}$, где $c_{1}=c_{1}(\lambda)$. Это следует из того, что по теореме Коши

$$
f(z)=\frac{1}{2 \pi i} \int_{\partial V_{n, j}} \frac{f(\zeta) d \zeta}{\zeta-z}+f_{n, j}(z),
$$

где $V_{n, j}$ - квадрат со стороной $\eta l_{n}, \eta=1 /(2 \lambda)>1$, концентрический с $E_{n, j}$, а $z$ достаточно близка к $\partial E_{n, j}$. Так как $\eta l_{n} \leqslant l_{n-1} / 2$, то $V_{n, j} \cap E_{n, i}=\varnothing, i \neq j$. Далее,

$$
\begin{aligned}
f_{n, j}^{\prime}(\infty) & =\frac{1}{2 \pi i} \int_{\gamma_{n, j}} f(z) d z=\frac{1}{2 \pi i} \int_{\gamma_{n, j}}\left(\int_{E_{n, j}} \frac{d \mu(\zeta)}{\zeta-z}\right) d z \\
& =\lim _{z \rightarrow \infty} z \int_{E_{n, j}} \frac{d \mu(\zeta)}{\zeta-z}=-a_{n, j} .
\end{aligned}
$$

Отсюда $\left|a_{n, j}\right| \leqslant 2 l_{n} / \pi$. Возьмем произвольную точку $z_{0} \in \mathbb{C}$. Пусть $j$ таково, что $\operatorname{dist}\left(z_{0}, E_{n, j}\right)=\min _{k} \operatorname{dist}\left(z_{0}, E_{n, k}\right)$. Так как $\sum_{k} f_{n, k}=f$, имеем

$$
\mathscr{C} \nu\left(z_{0}\right)=\varphi_{n, j}\left(z_{0}\right)+\sum_{k \neq j} g_{n, k}\left(z_{0}\right)+\sum_{k \neq j} f_{n, k}\left(z_{0}\right),
$$

где $g_{n, k}=-f_{n, k}+\varphi_{n, k}$. Поскольку

$$
\sum_{k \neq j} f_{n, k}(z)=f(z)-f_{n, j}(z)
$$

то

$$
\left|\sum_{k \neq j} f_{n, k}(z)\right|<c_{2}=c_{2}(\lambda), \quad z \in \mathbb{C} \backslash E_{n} .
$$

Значит, это неравенство выполняется и в точке $z_{0}$. Далее,

$$
\left|\varphi_{n, i}\left(z_{0}\right)\right|<\int_{0}^{l_{n} \sqrt{2}} \frac{\left|a_{n, i}\right|}{l_{n}^{2}} \frac{d \pi r^{2}}{r}<c_{3}, \quad i=1,2, \ldots, 4^{n} .
$$


Осталось оценить первую сумму в (14). Очевидно, $f_{n, k}(\infty)=\varphi_{n, k}(\infty)=0, f_{n, k}^{\prime}(\infty)=$ $\varphi_{n, k}^{\prime}(\infty)=-a_{n, k}$. Поэтому $g_{n, k}(\infty)=g_{n, k}^{\prime}(\infty)=0$. Кроме того, функция $g_{n, k}$ аналитична и ограничена по модулю некоторой постоянной $c_{4}=c_{4}(\lambda)$ вне квадрата $E_{n, k}$. По лемме Шварца [1, с. 13] имеем

$$
\left|g_{n, k}\left(z_{0}\right)\right| \leqslant c_{4} \frac{\operatorname{diam}\left(E_{n, k}\right) \gamma\left(E_{n, k}\right)}{\operatorname{dist}^{2}\left(z_{0}, E_{n, k}\right)} \leqslant c_{5} \frac{l_{n}^{2}}{\operatorname{dist}^{2}\left(z_{0}, E_{n, k}\right)} .
$$

Отсюда следует, что

$$
\sum_{k \neq j}\left|g_{n, k}\left(z_{0}\right)\right|<c_{6} l_{n}^{2}\left(\frac{4}{l_{n-1}^{2}}+\frac{16}{l_{n-2}^{2}}+\cdots+\frac{4^{n-1}}{l_{1}^{2}}\right)<c_{6} \sum_{i=1}^{\infty}\left(4 \lambda^{2}\right)^{i}=c_{7}
$$

где $c_{7}=c_{7}(\lambda)$. Лемма 1 доказана.

Нам потребуется понятие кривизны меры, введенное в [11, с. 58].

$K p$ ивизной $c(\mu)$ положительной борелевской меры $\mu$, заданной на плоскости $\mathbb{C}$, называется число, определяемое формулой

$$
c^{2}(\mu)=\iiint \frac{1}{R^{2}(x, y, z)} d \mu(x) d \mu(y) d \mu(z),
$$

где $R(x, y, z)$ - радиус окружности, проходящей через попарно различные точки $x, y, z$, и $1 / R^{2}(x, y, z)=0$, если $x=y$, или $x=z$, или $y=z$.

ДокАЗАТЕЛЬСТво ТЕОРЕмы 4. Рассмотрим некоторую положительную меру на множестве $E_{n}$, для которой $|\mathscr{C} \mu(z)|<1, z \in \mathbb{C} \backslash E_{n}$. Пусть $\nu$ - (положительная) мера из леммы 1. Тогда (см. [15], [16])

$$
\begin{aligned}
A^{2} \nu\left(E_{n}\right) & \geqslant \int_{E_{n}}\left|\int_{E_{n}} \frac{d \nu(x)}{x-y}\right|^{2} d \nu(y)=\int_{E_{n}}\left|\int_{E_{n}} \frac{d \nu(x)}{x-y}\right| \cdot\left|\int_{E_{n}} \frac{d \nu(z)}{\overline{z-y}}\right| d \nu(y) \\
& =\iiint_{E_{n}^{3}} \frac{1}{x-y} \frac{1}{\overline{z-y}} d \nu(x) d \nu(y) d \nu(z)=\frac{1}{6} c^{2}(\nu) .
\end{aligned}
$$

Положим $a_{k, j}=\nu\left(E_{k, j}\right)$. Покажем, что

$$
c^{2}(\nu)=\iiint_{E_{n}^{3}} \frac{1}{R^{2}(x, y, z)} d \nu(x) d \nu(y) d \nu(z)>c_{1} \sum_{k=1}^{n} \frac{1}{l_{k}^{2}} \sum_{j=1}^{4^{k}} a_{k, j}^{3} .
$$

Рассмотрим некоторый квадрат $E_{k, j}, 1 \leqslant k \leqslant n-1$. Из четырех квадратов $E_{k+1, i}$, входящих в $E_{k, j}$, выберем три, для которых величины $a_{k+1, i}$ наибольшие. Положим $S_{k, j}=\left\{(x, y, z) \in E_{k, j}^{(3)}: x, y, z\right.$ принадлежат трем попарно различным выбранным квадратам $\left.E_{k+1, i}\right\}, S_{n, j}=E_{n, j}^{(3)}$. Очевидно,

$$
c^{2}(\nu) \geqslant \sum_{k=1}^{n} \sum_{j=1}^{4^{k}} \iiint_{S_{k, j}} \frac{1}{R^{2}(x, y, z)} d \nu(x) d \nu(y) d \nu(z)
$$


Заметим, что при $(x, y, z) \in S_{k, j}, k<n$, вьполнено $R(x, y, z)<M l_{k}$, где $M=M(\lambda)$. Это следует из того, что $\lambda<1 / 3$. Назовем квадрат $E_{k, j}$ уравновешенны.м, если в нем найдутся такие три квадрата $E_{k+1, i}$, для каждого из которых $a_{k+1, i}>0.01 a_{k, j}$. Квадраты $E_{n, j}, j=1, \ldots, 4^{n}$, также будем считать уравновешенными. Нетрудно видеть, что если квадрат $E_{k, j}$ уравновешенный, то

$$
\iiint_{S_{k, j}} \frac{1}{R^{2}(x, y, z)} d \nu(x) d \nu(y) d \nu(z)>c_{2} \frac{a_{k, j}^{3}}{l_{k}^{2}} .
$$

Пусть теперь квадрат $E_{k, j}$ не является уравновешенньм. В него входят четыре квадрата $E_{k+1, i}$. Выделим тот из них, причем только один, для которого величина $a_{k+1, i}$ наибольшая, и обозначим его через $E_{k+1, i(j)}$. Покажем, что

$$
a_{k+1, i(j)} \geqslant 0.49 a_{k, j} \text {. }
$$

Действительно, не менее чем два из четырех квадратов $E_{k+1, i}$, входящих в $E_{k, j}$, имеют массу $a_{k+1, i} \leqslant 0.01 a_{k, j}$. Поэтому на остальные два квадрата $E_{k+1, i}$ из $E_{k, j}$ приходится масса, не меньшая чем $0.98 a_{k, j}$, откуда и следует (17).

Таким образом, каждый неуравновешенный квадрат входит в цепочку вложенных квадратов, притом только в одну:

$$
E_{k_{p}, j_{p}} \subset E_{k_{p-1}, j_{p-1}} \subset \cdots \subset E_{k_{1}, j_{1}},
$$

где $k_{t+1}=k_{t}+1, j_{t+1}=i\left(j_{t}\right), t=1, \ldots, p-1, p \geqslant 2$, причем квадрат $E_{k_{p}, j_{p}}$ уравновешенный, квадраты $E_{k_{t}, j_{t}}, t=1, \ldots, p-1$, неуравновешенные, и не существует неуравновешенного квадрата $E_{k_{1}-1, j}$, для которого $i(j)=j_{1}$. Используя $(16),(17)$, имеем

$$
\begin{gathered}
\sum_{t=1}^{p} \iiint_{S_{k_{t}, j_{t}}} \frac{1}{R^{2}(x, y, z)} d \nu(x) d \nu(y) d \nu(z)>\iiint_{S_{k_{p}, j_{p}}} \frac{1}{R^{2}(x, y, z)} d \nu(x) d \nu(y) d \nu(z) \\
\quad>c_{2} \frac{a_{k_{p}, j_{p}}^{3}}{l_{k_{p}}^{2}}=c_{3} \frac{a_{k_{p}, j_{p}}^{3}}{l_{k_{p}}^{2}} \sum_{N=0}^{\infty}\left(\frac{3^{-2}}{0.49^{3}}\right)^{N}>c_{3} \sum_{N=0}^{p-1} \frac{a_{k_{p-N}, j_{p-N}}^{3}}{l_{k_{p-N}}^{2}}=c_{3} \sum_{t=1}^{p} \frac{a_{k_{t}, j_{t}}^{3} .}{l_{k_{t}}^{2}}
\end{gathered}
$$

В последнем неравенстве мы воспользовались соотношениями

$$
\frac{a_{k_{p-N}, j_{p-N}}}{a_{k_{p}, j_{p}}} \leqslant \frac{1}{0.49^{N}}, \quad \frac{l_{k_{p}}}{l_{k_{p-N}}} \leqslant \lambda^{N}<\frac{1}{3^{N}} .
$$

Объединяя (16) и (18), получаем (15).

Заметим теперь, что

$$
\sum_{j=1}^{4^{k}} a_{k, j}=\nu\left(E_{n}\right), \quad 1 \leqslant k \leqslant n .
$$

При этом величина $\sum_{j=1}^{4^{k}} a_{k, j}^{3}$ достигает минимума, когда все слагаемые $a_{k, j}$ равны между собой, т.е. $a_{k, j}=\nu\left(E_{n}\right) 4^{-k}, j=1, \ldots, 4^{k}$. Поэтому

$$
\sum_{j=1}^{4^{k}} a_{k, j}^{3} \geqslant 4^{k}\left(\nu\left(E_{n}\right) 4^{-k}\right)^{3}=\left(\nu\left(E_{n}\right)\right)^{3} 4^{-2 k} .
$$


Отсюда и из (15) имеем

$$
A^{2} \nu\left(E_{n}\right)>\frac{c_{1}}{6}\left(\nu\left(E_{n}\right)\right)^{3} \sum_{k=1}^{n}\left(\frac{4^{-k}}{l_{k}}\right)^{2}
$$

т.e.

$$
\mu\left(E_{n}\right)=\nu\left(E_{n}\right)<c\left(\sum_{k=1}^{n}\left(\frac{4^{-k}}{l_{k}}\right)^{2}\right)^{-1 / 2},
$$

и теорема 4 доказана.

ЛЕмма 2. Пусть измеряющая функиия $h$ удовлетворяет условию (8) с некоторыми постоянными $r_{0}>0, b \in(0,1) u \alpha \in\left(0, \log _{3} 4\right)$. Тогда для любого $l_{0} \in\left(0, r_{0}\right]$ существуют числа $l_{j}>0$ такие, что $l_{j} \leqslant \lambda l_{j-1}, j \geqslant 1$, c $\lambda=4^{-1 / \alpha}<1 / 3, u$

$$
b 4^{-j} h\left(l_{0}\right) \leqslant h\left(l_{j}\right) \leqslant 4^{-j} h\left(l_{0}\right) .
$$

ДокАЗАТЕЛЬСТво. Положим $N_{0}=0$. Пусть при некотором $i \geqslant 0$ числа $N_{1}, \ldots, N_{i}$ уже определены, и при некотором $j>N_{i}$ числа $l_{1}, \ldots, l_{j-1}$ построены, причем

$$
h\left(l_{N_{i}}\right)=4^{-N_{i}} h\left(l_{0}\right)
$$

Возможны два случая.

1) $h\left(\lambda l_{j-1}\right) \geqslant 4^{N_{i}-j} h\left(l_{N_{i}}\right)$. Тогда полагаем $N_{i+1}=j$ и величину $l_{j}$ определяем из равенства $h\left(l_{j}\right)=4^{N_{i}-j} h\left(l_{N_{i}}\right)$. Ясно, что $l_{j} \leqslant \lambda l_{j-1}$, вьполнено условие (19) и имеет место (20) с индексом $i+1$ вместо $i$.

2) $h\left(\lambda l_{j-1}\right)<4^{N_{i}-j} h\left(l_{N_{i}}\right)$. Тогда полагаем $l_{j}=\lambda l_{j-1}$. Из $(20)$ следует, что второе неравенство в (19) вьполнено. Поскольку $l_{j}=\lambda l_{j-1}$ при $N_{i}+1 \leqslant j<N_{i+1}$, то $l_{j} / l_{N_{i}}=\lambda^{j-N_{i}}$. Отсюда в силу (8) и (20) имеем

$$
h\left(l_{j}\right) \geqslant b h\left(l_{N_{i}}\right)\left(\frac{l_{j}}{l_{N_{i}}}\right)^{\alpha}=b 4^{-N_{i}} h\left(l_{0}\right) \lambda^{\alpha\left(j-N_{i}\right)}=b 4^{-N_{i}} h\left(l_{0}\right) 4^{-\left(j-N_{i}\right)}=b 4^{-j} h\left(l_{0}\right),
$$

что и требовалось доказать.

ДоКАЗАТЕЛЬСТво ТЕОРЕмЫ 3 . Возьмем последовательность $\left\{l_{j}\right\}$, существование которой утверждается в лемме 2 , и соответствуюшее канторово множество $E=E\left(\left\{l_{j}\right\}\right)$. Покажем, что множество $E$ является искомым. Используя (19), имеем

$$
\begin{aligned}
\sum_{k=1}^{\infty}\left(\frac{4^{-k}}{l_{k}}\right)^{2} & =\frac{1}{16} \sum_{k=1}^{\infty}\left(\frac{4^{-(k-1)}}{l_{k}}\right)^{2} \geqslant \frac{1}{16 h\left(l_{0}\right)} \sum_{k=1}^{\infty}\left(\frac{h\left(l_{k-1}\right)}{l_{k}}\right)^{2} \\
& \geqslant c \sum_{k=1}^{\infty} \int_{l_{k}}^{l_{k-1}} \frac{d h^{2}(t)}{t^{2}}=c \int_{0}^{l_{0}} \frac{d h^{2}(t)}{t^{2}}=\infty
\end{aligned}
$$

поскольку интегралы $\int_{0} t^{-3} h^{2}(t) d t$ и $\int_{0} t^{-2} d h^{2}(t)$ сходятся или расходятся одновременно. Отсюда и из $(9)$ следует, что $\gamma^{+}(E)=0$. Доказательство $h$-регулярности множества $E$ проводится так же, как и в следствии 1, с использованием неравенств (13) и (19).

Доказательство предложения 1 основьвается на следующей теореме. 
Teорема $7[11$, с. 59, теорема 3]. Для любого компакта $E \subset \mathbb{C}$

$$
\gamma(E) \geqslant P \cdot \sup \left\{(\mu(E))^{3 / 2}\left(\mu(E)+c^{2}(\mu)\right)^{-1 / 2}\right\},
$$

где $P$ - абсолютная постоянная и супремум берется по всем положительным борелевским мерам $\mu$ таким, что $\operatorname{supp} \mu \subset E u \mu(B(z, r)) \leqslant r$ для любого диска $B(z, r)$.

ДоКАЗАТЕЛЬСТВо ПРЕДЛОЖЕНИЯ 1 . Пусть Задано $r<l_{0}$ и число $k$ таково, что $l_{k} \leqslant r<l_{k-1}$. Тогда каждый круг радиуса $r$ содержит не более чем $A$ квадратов $E_{k, j}$, где $A>4-$ абсолютная постоянная. Обозначим

$$
M=\frac{1}{A}\left(\sum_{j=1}^{n}\left(\frac{4^{-j}}{l_{j}}\right)^{2}\right)^{-1 / 2} .
$$

В качестве $\mu$ возьмем меру такую, что $\mu\left(E_{n, j}\right)=4^{-n} M, j=1, \ldots, 4^{n}$, которая на каждом квадрате $E_{n, j}$ распределена равномерно. Очевидно, $\mu(E)=M$. При $r<l_{0}$ и $k$ таком, что $l_{k} \leqslant r<l_{k-1}$, имеем

$$
\mu_{z}(r):=\mu(B(z, r)) \leqslant A M 4^{-k}<l_{k} \leqslant r .
$$

При $r \geqslant l_{0}$ будет $\mu_{z}(r) \leqslant M<l_{1}<r$, и условие теоремы 7 выполнено. на $\mathbb{C}$

В $[8$, c. 198$]$ показано, что для любой конечной положительной борелевской меры $\mu$

$$
c^{2}(\mu) \leqslant 12 \int_{\mathbb{C}} \int_{0}^{\infty} \frac{\mu_{z}(r)}{r^{2}} d \mu_{z}(r) d \mu(z) .
$$

Для рассматриваемой меры $\mu$ имеем

$$
\begin{aligned}
\int_{0}^{\infty} \frac{\mu_{z}(r)}{r^{2}} d \mu_{z}(r) & =\int_{0}^{l_{n}} \frac{\mu_{z}(r)}{r^{2}} d \mu_{z}(r)+\sum_{k=1}^{n} \int_{l_{k}}^{l_{k-1}} \frac{\mu_{z}(r)}{r^{2}} d \mu_{z}(r)+\int_{l_{0}}^{\infty} \frac{\mu_{z}(r)}{r^{2}} d \mu_{z}(r) \\
& <\pi^{2} M^{2}\left(\frac{4^{-n}}{l_{n}}\right)^{2}+A^{2} M^{2} \sum_{k=1}^{n}\left(\frac{4^{-k}}{l_{k}}\right)^{2}+\frac{M^{2}}{l_{0}^{2}}<A_{1}
\end{aligned}
$$

и в силу $(22) c^{2}(\mu) \leqslant A_{2} M$. Из теоремы 7 получаем неравенство $\gamma(E)>A_{3} M$, что и требовалось.

Автор признателен факультету математики университета Уппсалы (Швеция) за прекрасные условия, в которых было начато данное исследование, а профессору М. Эссену за гостеприимство и поддержку этого начинания. Я благодарен также профессору П. Маттиле и профессору С. Я. Хавинсону за очень полезное обсуждение данной проблематики.

ДОБАВЛЕНИЕ ПРИ КОРРЕКТУРЕ. В апреле 1998 года перед выходом данной работы профессор М. С. Мельников сообщил автору о диссертации Х. Толсы [17]. В этой работе показано, в частности, что имеет место усиленная версия теоремы 7 М. С. Мельникова: вместо $\gamma(E)$ можно взять $\gamma^{+}(E)$. При этом согласно теореме 6 для $\mu$ и $h$, удовлетворяющих условиям (4) и (11), выполнено $c^{2}(\mu)>0$. Следовательно, в теореме 5 также можно взять $\gamma^{+}$вместо $\gamma$. Отсюда сразу получается следующее усиление теорем 1 и 2 : в условиях теоремы 2 вьполнено $\gamma^{+}(E)>0$. Если применить результат работы [17] 
при доказательстве предложения 1 , то получится неравенство $(10)$ с $\gamma^{+}$вместо $\gamma$. Тем самым, первое неравенство в гипотезе 2 оказьвается доказанным. Вопрос о справедливости второго неравенства остается открытым. В [17] получено также другое доказательство следствия 2.

Автор благодарен М. С. Мельникову и Х. Толсе за информацию о работе [17] и обсуждение ряда открытых вопросов.

\section{СПИСОК ЦИТИРОВАННОЙ ЛИТЕРАТУРЫ}

[1] Garnett J. Analytic Capacity and Measure. Lecture Notes in Math. V. 297. Berlin-Heidelberg-New York: Springer, 1972.

[2] Murai T. A Real Variable Method for the Cauchy Transform, and Analytic Capacity. Lecture Notes in Math. V. 1307. Berlin: Springer, 1988.

[3] Карлесон Л. Избранные проблемы теории исключительных множеств. М.: Мир, 1971.

[4] Эйдерман В.Я. О сравнении меры Хаусдорфа и емкости // Алгебра и анализ. 1991. Т. 3. №6. C. $173-188$.

[5] Эйдерман В.Я. Оценки потенциалов и $\delta$-субгармонических функций вне исключительных множеств // Изв. РАН. Сер. матем. 1997. Т. 61. №6. С. 181-218.

[6] Витушкин А. Г. Пример множества положительной длины и аналитической емкости нуль // Докл. АН СССР. 1959. Т. 127. № 2. С. 246-249.

[7] Garnett J. Positive length but zero analytic capacity // Proc. Amer. Math. Soc. 1970. V. 24. P. 696-699.

[8] Mattila P. A class of sets with positive length and zero analytic capacity // Ann. Acad. Sci. Fenn. Ser. A. I Math. 1985. V. 10. P. 387-395.

[9] Havin V.P., Nikolski N. K. (ed. ) Linear and Complex Analysis Problem Book 3. Part 2. Lecture Notes in Math. V. 1574. Berlin: Springer, 1994.

[10] Mattila P. On the analytic capacity and curvature of some Cantor sets with non- $\sigma$-finite length // Publ. Mat. 1996. V. 40. №1. P. 195-204.

[11] Мельников М. С. Аналитическая емкость: дискретный подходи кривизна меры // Матем. сб. 1995. Т. 186. №6. С. 57-76.

[12] Christ M. Lectures on Singular Integral Operators. Expository Lectures from the CBMS Regional Conference Held at the University of Montana, Missoula (M.T.), August 28-September 1, 1989. CBMS Regional Conf. Ser. in Math. V. 77. Providence (R.I.): Amer. Math. Soc., 1990.

[13] Eiderman V. Ya. Analytic capacity of Cantor sets // Proceedings of the International Conference on Potential Theory (Kouty, Czech Republic, 1994) / ed. I. Netuka et al. Berlin-New York: Walter de Gruyter, 1995.

[14] Murai T. Construction of $H^{1}$ functions concerning the estimate of analytic capacity // Bull. London Math. Soc. 1987. V. 19. P. 154-160.

[15] Melnikov M.S., Verdera J. A geometric proof of the $L^{2}$-boundedness of the Cauchy integral on Lipschitz graphs // Internat. Math. Res. Notices. 1995. № 7. P. 325-331.

[16] Mattila P., Melnikov M.S., Verdera J. The Cauchy integral, analytic capacity, and uniform rectifiability // Ann. of Math. (2). 1996. V. 144. №1. P. 127-136.

[17] Tolsa X. Curvature of Measures, Cauchy Singular Integral and Analytic Capacity. Thesis. Barcelona: Departament de Matemàtiques, Universitat Autònoma de Barcelona, 1998.

Московский государственный строительный университет E-mail : eiderman@orc.ru 